\title{
Analysis of enteric disease outbreak metrics, British Columbia Centre for Disease Control, 2005-2014
}

\author{
D Fong ${ }^{1,2^{\star}}$, M Otterstatter ${ }^{1,3}$, M Taylor ${ }^{1}$, E Galanis ${ }^{1,3}$
}

\section{Abstract}

Background: For enteric disease outbreaks, effective control depends on timely intervention. Routine collection of metrics related to outbreak identification, investigation and control can help evaluate and improve interventions and inform further analyses and modelling of intervention effectiveness.

Objective: To analyze data from enteric disease outbreaks in British Columbia, generate outbreak metrics and assess their use in evaluating the impact of outbreak interventions.

Methods: This descriptive study analyzed data from 57 provincial and national enteric disease outbreak investigations involving the British Columbia Centre for Disease Control from 2005 to 2014. Data were extracted from internal files and the Canadian Network for Public Health Intelligence. Outbreak metrics analyzed included days to initiate investigation, days to intervention, number and type of interventions, duration of investigation, duration of outbreak and the total number of cases.

Results: The median time to initiate an outbreak investigation was 36 days and the median duration of investigations was 39 days. The median duration of outbreaks was 40 days and the median time to intervene was 10 days. Identification of the source was associated with use of one or more interventions $(P<0.0001)$. The duration of outbreaks was correlated with the number of days to initiate an investigation $\left(r_{s}=0.72, P<0.0001\right)$ and number of days to intervene $\left(r_{s}=0.51, P=0.025\right)$.

Conclusion: Identification and analysis of outbreak metrics establishes benchmarks that can be compared to other jurisdictions. This may support continuous quality improvement and enhance understanding of the impact of public health activities. Date information for public health actions is essential for evaluating the timing and effectiveness of outbreak interventions.
Affiliations

${ }^{1}$ British Columbia Centre for Disease Control, Vancouver, BC

${ }^{2}$ National Collaborating Centre for Environmental Health, Vancouver, BC

${ }^{3}$ School of Population and Public Health, University of British Columbia, Vancouver, BC

*Correspondence: daniel.fong@ bccdc.ca

Suggested citation: Fong D, Otterstatter M, Taylor M, Galanis E. Analysis of enteric disease outbreak metrics, British Columbia Centre for Disease Control, 2005-2014. Can Commun Dis Rep. 2017;43(1):1-6. https://doi.org/10.14745/ccdr.v43i01a01

\section{Introduction}

The objective of an outbreak investigation is to identify the source and implement timely and appropriate interventions to control the outbreak (1). The timeliness of control measures often depends on how quickly an outbreak is first identified and solved. If implementation of control measures is delayed, they may have little impact. Hence, it is of value to track metrics (indicators that can be compared over time and to other jurisdictions) related to the timeliness of outbreak identification, investigation and control. Historical outbreak data can be used to create these measures.

Routine collection and analysis of outbreak metrics can inform quality improvement activities. In the United States of America, the Foodborne Diseases Centers for Outbreak Response Enhancement (FoodCORE) program collects standardized metrics on foodborne disease outbreaks to improve outbreak response (2). Outbreak data have also been used to assess the impact of interventions. Seto et al. (2007) used data from a multistate outbreak of Escherichia coli O157:H7 in the United States to model control strategies and found that reducing secondary transmission by $1-25 \%$ could prevent $2-3 \%$ of secondary cases and $5-11 \%$ of infected and symptomatic individuals (3). Chen et al. (2014) used data from a waterborne shigellosis outbreak at a school in China to examine the effect and optimal combination of five interventions on the attack rate and outbreak duration (4). Despite the usefulness of outbreak metrics, such measures are seldom evaluated or reported (5).

The British Columbia Centre for Disease Control (BCCDC) is responsible for coordinating investigations of enteric disease outbreaks that affect more than one region in British-Columbia (BC). It also assists in investigating outbreaks that affect a single 
$\mathrm{BC}$ region or the province and multiple Canadian provinces. This involves identifying outbreaks, developing case definitions and outbreak questionnaires, analyzing the epidemiologic data collected, implementing epidemiologic studies, providing recommendations on control measures and communicating with the public (6). Information resulting from these activities can be used to improve public health interventions and their impact. Efforts should be made to develop and measure indicators that are useful to public health partners and the general public.

In this article, we present the first phase of a study to assess the impact of interventions on the duration and size of enteric disease outbreaks. The objective of this study was to analyze British Columbia enteric disease outbreak data, generate outbreak metrics and assess their use in evaluating the impact of outbreak interventions.

\section{Methods}

Data from provincial and national enteric disease outbreak investigations that involved the BCCDC were included for analysis. Data from 2008 to 2014 were extracted from the Canadian Network for Public Health Intelligence (CNPHI) on July 24, 2015. Data on outbreaks involving the BCCDC from 2005 to 2008 were extracted from internal files at the BCCDC, including outbreak summaries and investigation meeting minutes.

\section{Outbreak inclusion criteria}

We defined BCCDC involvement based on two criteria: at least one of the enteric illness cases was in British Columbia and BCCDC participated in the outbreak investigation through meetings, by providing epidemiologic support and/or by coordinating the investigation of the outbreak. Enteric outbreaks were included if they met the definition of community outbreak ( $\geq 2$ unrelated cases with similar illness that are epidemiologically linked), institution outbreak ( $\geq 3$ cases with similar illness that are epidemiologically linked) or a single case of botulism (based on the Public Health Agency of Canada's Outbreak Summaries User Manual version 2).

\section{Outbreak metrics}

Where applicable, outbreak metrics were defined using the Guidelines for Foodborne Disease Outbreak Response developed by the Council to Improve Foodborne Outbreak Response (1). Otherwise, definitions are consistent with those in the CNPHI data dictionary (Government of Canada. Outbreak Summaries Data Dictionary - Enteric, Food and Water Borne Disease Module. Canadian Network for Public Health Intelligence. n.d.).

Where available, we extracted or calculated the outbreak metrics defined in Table 1.

Time-related outbreak metrics are consistent with events of a typical enteric disease outbreak (Figure 1). Other variables such as number and type of interventions implemented, etiologic agent, mode of transmission, source details, location of cases, and reporting agency were used to provide context to the results.

\section{Table 1: Definitions of key enteric outbreak metrics}

\begin{tabular}{|l|l|}
\hline \multicolumn{1}{|c|}{$\begin{array}{c}\text { Outbreak } \\
\text { metric }\end{array}$} & \multicolumn{1}{c|}{ Definition } \\
\hline $\begin{array}{l}\text { Days to initiate } \\
\text { investigation }\end{array}$ & $\begin{array}{l}\text { Interval between date of earliest known symptom } \\
\text { and start of outbreak investigation }\end{array}$ \\
\hline $\begin{array}{l}\text { Days to } \\
\text { intervene }\end{array}$ & $\begin{array}{l}\text { Interval between date of start of outbreak } \\
\text { investigation and date of implementing first } \\
\text { outbreak intervention, if any }\end{array}$ \\
\hline $\begin{array}{l}\text { Duration of } \\
\text { investigation }\end{array}$ & $\begin{array}{l}\text { Interval, in days, between start and end dates of } \\
\text { outbreak investigation }\end{array}$ \\
\hline $\begin{array}{l}\text { Duration of } \\
\text { outbreak }\end{array}$ & $\begin{array}{l}\text { Interval, in days, between date of onset of earliest } \\
\text { known symptom and date of lastest symptom } \\
\text { onset date }\end{array}$ \\
\hline $\begin{array}{l}\text { Total number of } \\
\text { cases }\end{array}$ & $\begin{array}{l}\text { Total number of reported clinical and lab-confirmed } \\
\text { cases in outbreak }\end{array}$ \\
\hline
\end{tabular}

An intervention was defined as public health action intended to eliminate or decrease exposure to the source of an outbreak or decrease an individual's susceptibility to infection. Types of interventions included actions on facilities (closure, staff exclusion, sanitization) as well as education, immunization, policy changes, press releases and product recalls.

Figure 1: Progression of an enteric disease outbreak and time-related outbreak metrics

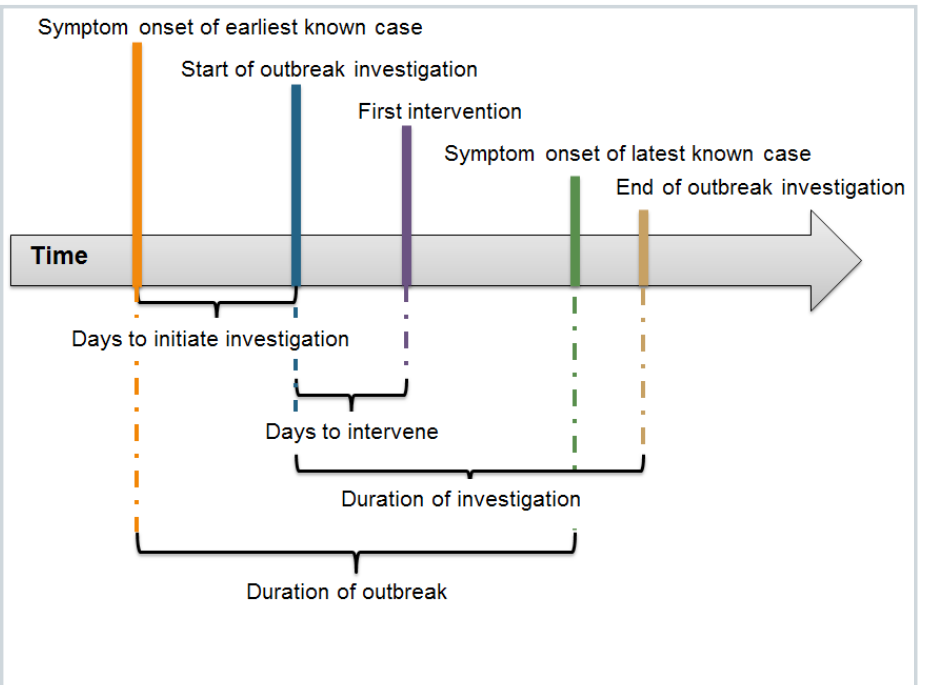

Outbreaks were described using counts of cases and days, as well as medians and ranges. Inferential analyses were conducted to uncover preliminary relationships between outbreak investigation activities, the timing of those activities and/or case counts; this was done to inform approaches to evaluate intervention effectiveness in future studies. Fisher's exact test was used to test for statistical significance of relationship between knowledge of outbreak source and use of interventions. Spearman's rank correlation was used to test for statistical significance of relationships between time-related 
outbreak metrics (e.g. durations) and case counts. Analyses were performed using statistical package R version 3.2.2 and Microsoft Excel 2010.

\section{Results}

\section{Outbreak metrics}

Characteristics of the outbreaks are summarized in Table 2. From a total of 57 enteric outbreaks involving the BCCDC from 2005 to 2014 , the majority $(88 \%, n=50)$ had cases located in more than one regional health authority and most $(79 \%, n=45)$ were foodborne. The median number of cases per outbreak of Salmonella, E. coli O157:H7 and overall were 22.5 (range: 3-1029), 16 (range: 3-85) and 18 (range: 1-1029), respectively. Salmonella and E. coli O157:H7 were implicated in $63 \%(n=36)$ of outbreaks and contributed to $76 \%(n=2291)$ of the outbreak-related cases. During the study period, there was a median of six outbreaks investigated per year (range: 3-8) with nearly half $(47 \%, n=27)$ occurring in the summer (June to August). The source was identified in $46 \%(n=26)$ of outbreaks.

Table 2: Characteristics of enteric outbreaks involving the British Columbia Centre for Disease Control, by etiology, 2005-2014

\begin{tabular}{|c|c|c|c|c|c|}
\hline \multirow{2}{*}{\multicolumn{2}{|c|}{ Outbreak metric }} & Salmonella & $\begin{array}{c}\text { E. coli } \\
\text { 0157:H7 }\end{array}$ & Other ${ }^{1}$ & \multirow[t]{2}{*}{$\begin{array}{l}\text { Total } \\
\text { N (\%) }\end{array}$} \\
\hline & & N (\%) & N (\%) & N (\%) & \\
\hline \multicolumn{2}{|l|}{ Outbreaks } & $24(42)$ & $12(21)$ & $21(37)$ & $57(100)$ \\
\hline \multicolumn{2}{|l|}{ Cases } & 2025 (67) & $266(9)$ & $742(25)$ & $3033(100)$ \\
\hline \multirow{4}{*}{ Location } & International & $7(12)$ & $3(5)$ & $1(2)$ & $11(19)$ \\
\hline & $\begin{array}{l}>1 \text { province/ } \\
\text { territory }\end{array}$ & $9(16)$ & $8(14)$ & $8(14)$ & $25(44)$ \\
\hline & $>1 \mathrm{HU} / \mathrm{RHA}$ & $8(14)$ & $1(2)$ & $5(9)$ & $14(25)$ \\
\hline & $1 \mathrm{HU} / \mathrm{RHA}$ & 0 & 0 & $7(12)$ & $7(12)$ \\
\hline \multirow{3}{*}{$\begin{array}{l}\text { Mode of } \\
\text { transmission }\end{array}$} & Foodborne & $18(32)$ & $10(18)$ & $17(30)$ & 45 (79) \\
\hline & Other $^{2}$ & $3(5)$ & 0 & $1(2)$ & $4(7)$ \\
\hline & Unknown & $3(5)$ & $2(4)$ & $3(5)$ & $8(14)$ \\
\hline \multirow{2}{*}{ Source } & Known ${ }^{3}$ & $12(21)$ & $6(11)$ & $8(14)$ & $26(46)$ \\
\hline & Unknown & $12(21)$ & $6(11)$ & $13(23)$ & $31(54)$ \\
\hline \multirow[t]{2}{*}{ Interventions } & $\begin{array}{l}>1 \\
\text { intervention }\end{array}$ & $14(25)$ & $6(11)$ & $7(12)$ & $27(47)$ \\
\hline & Total & $31(54)$ & $12(21)$ & $14(25)$ & $57(100)$ \\
\hline
\end{tabular}

Abbreviations: N, number; HU/RHA, Health Unit/Regional Health Authority; \%, percentage ${ }^{1}$ Cyclospora $(n=5)$, Clostridium botulinum $(n=4)$, Campylobacter $(n=3)$, hepatitis A virus $(n=3)$ shellfish poisoning (diarretic/paralytic, $n=2)$, Shigella $(n=2)$, Listeria $(n=1)$ and norovirus $(n=1)$ ${ }^{2}$ Animal-to-person, person-to-person or contaminated pet treats

${ }^{3}$ Sources identified were primarily food $(42.1 \%, \mathrm{n}=24)$ including meat, vegetables/fruits, seafood, eggs, condiments, seed/nuts/legumes and dairy. Pet treats were implicated in 2 (3.5\%) outbreaks

Time-related outbreak metrics are summarized in Table 3. Outbreak data were generally available for calculating days to initiate investigation ( $93 \%$ complete, $n=53$ ), duration of investigation $(77 \%$ complete, $n=44)$ and duration of each outbreak ( $95 \%$ complete, $n=54$ ). The median time to start an outbreak investigation was 36 days and the median duration of investigations was 39 days. Outbreaks with short times to investigation were less widespread geographically. For example,
$85 \%(n=11 / 13)$ of the investigations initiated within 18 days $\left(1^{\text {st }}\right.$ quartile) involved only one province, whereas $92 \%(n=11 / 12)$ of the investigations initiated after 55 days ( $3^{\text {rd }}$ quartile) had cases in more than one province/territory. In addition, outbreaks with short times to investigation were those with distinct symptoms or etiologic agents with short incubation periods or those that did not require molecular subtyping for links to be established (e.g. paralytic shellfish poisoning, Clostridium botulinum and norovirus). In contrast, etiologic agents with long incubation periods, such as hepatitis A virus and Listeria, were associated with investigations that took longer than the median 36 days to initiate.

Table 3: Time-related enteric outbreak metrics involving the British Columbia Centre for Disease Control, 2005-2014

\begin{tabular}{|l|r|r|r|r|r|}
\hline \multicolumn{1}{|c|}{$\begin{array}{c}\text { Outbreak } \\
\text { metric }\end{array}$} & Median & \multicolumn{1}{|c|}{$\begin{array}{c}1^{\text {st }} \\
\text { quartile }\end{array}$} & $\begin{array}{r}3^{\text {rd }} \\
\text { quartile }\end{array}$ & Range & N \\
\hline $\begin{array}{l}\text { Days to initiate } \\
\text { investigation }\end{array}$ & 36 & 18 & 55 & $0-620$ & 53 \\
\hline $\begin{array}{l}\text { Duration of } \\
\text { investigation } \\
\text { (days) }\end{array}$ & 39 & 20 & 78 & $0-1651$ & 44 \\
\hline $\begin{array}{l}\text { Duration of } \\
\text { outbreak (days) }\end{array}$ & 10 & 16 & 84 & $0-1689$ & 54 \\
\hline $\begin{array}{l}\text { Days to } \\
\text { intervene, first } \\
\text { intervention }\end{array}$ & 6 & 4 & 33 & $2-140$ & 14 \\
\hline $\begin{array}{l}\text { Days to press } \\
\text { release }\end{array}$ & 13 & 10 & 25 & $1-74$ & 6 \\
\hline $\begin{array}{l}\text { Days to product } \\
\text { recall }\end{array}$ & & 4 & 19 & $2-106$ & 16 \\
\hline $\begin{array}{l}\text { Days to } \\
\text { intervene, other } \\
\text { interventions }\end{array}$ & & & & & 6 \\
\hline
\end{tabular}

Abbreviation: N, Number

${ }^{1}$ Close facility, education, exclude staff, immunize susceptibles

\section{Outbreak intervention metrics}

Almost half $(47 \%, n=27)$ of the outbreaks had at least one recorded intervention, with most of these $(70 \%, n=19)$ having no more than two interventions (Table 2). The median number of interventions per outbreak for Salmonella, E. coli O157:H7 and overall were 1 (range: $0-5$ ), 0.5 (range: $0-3$ ) and 0 (range: $0-5)$, respectively. Out of a total of 57 interventions documented, most $(75 \%, n=43)$ were associated with Salmonella or E. coli 0157:H7. Identification of the source was significantly associated with use of one or more interventions $(P<0.0001)$. Product recall, facility closure, facility sanitization and immunization were only implemented when a source was identified, whereas press releases, education, staff exclusion and policy changes were implemented irrespective of whether the source was identified (Figure 2). Besides product recalls and press releases, other intervention types were reported infrequently or their implementation dates were unavailable; 21 (37\%) of 57 interventions had insufficient information to calculate the time 
to intervene. Of the 27 outbreaks with at least one intervention, $74 \%(n=20)$ had sufficient information to calculate time to intervene. The median time to intervene was 10 days, with product recalls implemented a median of nine days after the start of an outbreak investigation and press releases a median of six days after the start of an outbreak investigation (Table 3).

Figure 2: Frequency and type of interventions used, according to whether the outbreak source was known $(n=57)$

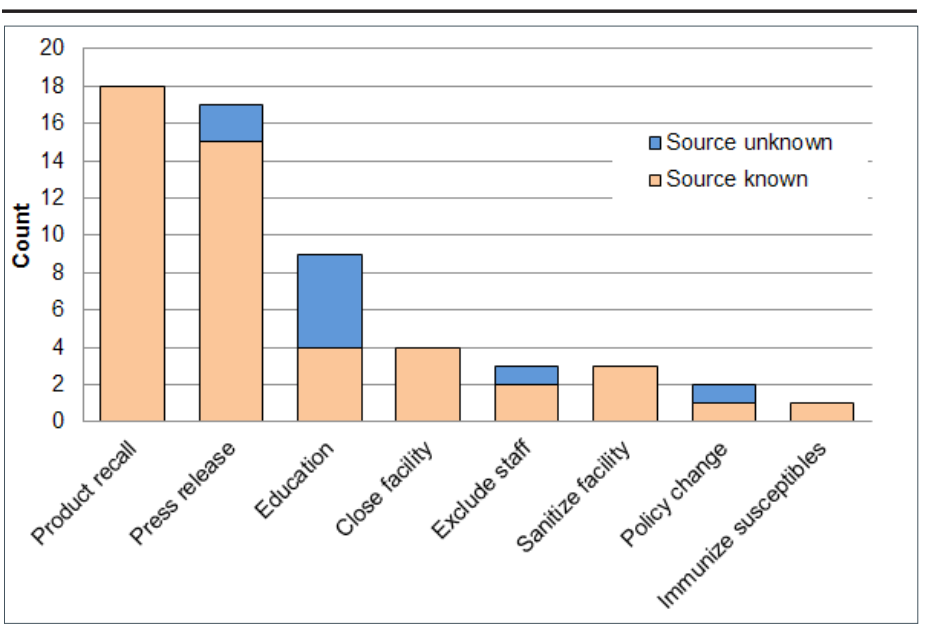

The duration of outbreaks was positively correlated with the number of days to initiate an investigation $\left(r_{s}=0.72, P<0.0001\right)$ and number of days to intervene $\left(r_{s}=0.51, P=0.025\right)$. The number of days to intervene was also positively correlated with the number of days to initiate an investigation $\left(r_{s}=0.47, P=0.036\right)$. There were no significant correlations between the total number of cases and the number of days to initiate an investigation or days to intervene $\left(r_{s}=0.16, P=0.27 ; r_{s}=-0.082, P=0.73\right.$, respectively).

\section{Discussion}

At the time of this study, we were not aware of any published analyses of metrics related to enteric disease outbreaks and associated interventions in Canada. We provide information on outbreak metrics that can be used to assess the timing of enteric disease outbreak investigation and intervention.

\section{Implementing interventions}

Our findings indicate that identifying the source of an outbreak was associated with implementing interventions. For certain interventions (e.g. product recall), source identification is required as these interventions eliminate the implicated source. Non-specific interventions (e.g. education) can be implemented without knowledge of a definitive source. We found that product recalls, press releases and education were used more often than facility closures and exclusion of ill persons, which is consistent with the fact that our outbreaks tended to be widespread rather than localized or facility-based. FoodCORE found that, between 2010 and 2012, a source was identified in an average of $30 \%$ of Salmonella, E. coli O157:H7 and Listeria outbreaks (2). In our study, almost half the outbreaks had a known source (Table 2). This difference is possibly due to more stringent criteria for initiating outbreak investigations in British Columbia since 2011, established to focus on those outbreaks whose source may be more easily identified (7). Source identification is a critical milestone for implementing targeted control actions.

\section{Outbreak duration and total cases}

Our findings indicate that as the number of days to initiate an investigation increases, so does the duration of outbreak and number of days to intervene. Similarly, as the number of days to initiating intervention increases, so too does the duration of the outbreak. Where interventions are delayed, disease transmission will continue and lead to longer outbreaks. Our preliminary findings indicate a correlation between timing of outbreak activities and outbreak duration. Further research is required to substantiate these relationships.

Regular tracking of outbreak metrics allows comparison against internal and external benchmarks over time and identification of activities for improvement. FoodCORE has been reporting annual outbreak metrics since 2011 for evaluation of outbreak detection, response and control activities (8-11). They report a median duration of investigation (calculated from cluster notification to end of investigation) for Salmonella of 26 to 35 days (12-14); based on our data, we found this median duration of investigation to be 49 days. This difference may be due to our dataset including several large outbreaks (>100 days), including an S. Enteritidis outbreak that spanned four years. Operational differences in outbreak response and surveillance activities may be another reason for the differences in reported duration. Such inherent differences in data sources and operations may limit external comparisons, but internal comparisons over time are valuable for tracking improvements. Collecting outbreak metrics and reporting on how they are used as performance indicators will allow for evaluation and richer analysis of trends, including those for intervention timing.

Although our analysis did not find a significant relationship, one might expect the total number of cases to decrease when investigations or interventions are initiated earlier. One review of European outbreak investigations found no correlation between the timeliness of completing an analytic outbreak study and the total number of cases (5). Still, models have indicated that delays in reporting of the index case to public health increases the proportion of expected infections produced by index and secondary cases (15). Case counts are likely influenced by other factors related to transmission dynamics.

\section{Data limitations and implications for future research}

The absence of date information for many interventions limits the ability to conduct further analysis. The pooled data are skewed by the large proportion of Salmonella and E. coli O157:H7 outbreaks; summary statistics could not be calculated for many other etiologic agents.

Although outbreak investigations may follow a logical sequence of activities, our data were largely cross-sectional in nature, which limits specific inferences about cause and effect. Case counts and time-related outbreak metrics varied substantially and statistical power was limited by a small sample size. Outbreaks reported by regional health authorities that did not involve the BCCDC 
were excluded as their scope is operationally distinct (e.g. different settings, management) and required data that were not accessible by the BCCDC. Therefore, the results reported may only apply to situations involving widespread outbreaks (provincial or national).

Since 2011, the BCCDC has established criteria that consider the minimum number and geographic distribution of cases for initiating an enteric outbreak investigation (7). In our results, we see this reflected in the large proportion of foodborne Salmonella and E. coli O157:H7 multi-jurisdictional outbreaks and small proportion of localized outbreaks from etiological agents with shorter incubation periods primarily spread from person to person (e.g. norovirus). Therefore, any time-related outbreak metrics and interventions should be interpreted in the context of the type of outbreaks included in the analysis.

Thorough and consistent documentation of outbreaks, including complete line lists and dates for outbreak milestones would be needed to further explore the impact of interventions. Additional data and mathematical modelling would be useful for exploring the relationships among intervention timing, duration of outbreaks, the number of cases over time and the expected number of cases averted. Modelling may also permit assessment of combined intervention strategies, which more closely reflects real-world situations. Based on data from previous outbreaks, studies have used models to simulate the expected temporal distribution of cases (epidemic curve) and quantify the effect of interventions $(3,4)$.

\section{Conclusion}

This study describes how outbreak data can be used to develop outbreak metrics and use them to evaluate the timing of investigations and interventions. We identified outbreak metrics suitable for establishing baselines. These findings will help determine methodological and data quality considerations for future studies to predict the impact of interventions on outbreak duration and case counts over time. Temporal information of key outbreak milestones is essential. Routine analysis of outbreak data may help identify requirements for action, establish benchmarks, support continuous quality improvement and enhance understanding of the impact of public health activities on the outcomes of an outbreak. We encourage partnerships between agencies to address data gaps and develop evidence-informed approaches to assess the utility of outbreak response and control actions.

\section{Acknowledgements}

We would like to thank Dr. Jane Buxton for her critical review of the manuscript. We also acknowledge the environmental health officers in British Columbia and across Canada for reporting outbreaks through the Canadian Network for Public Health Intelligence and the local and provincial laboratories for their diagnostic work.

\section{Conflict of interest}

None.

\section{Funding}

This work was supported in kind by the BCCDC and the University of British Columbia.

\section{References}

1. Council to Improve Foodborne Outbreak Response (CIFOR). Guidelines for foodborne disease outbreak response [Internet]. Atlanta: Council of State and Territorial Epidemiologists; 2014 [cited 2016 Nov 4]. Available from: http://www.cifor.us/documents/CIFOR\%20Industry\%20 Guidelines/CIFOR-Industry-Guideline.pdf.

2. Biggerstaff GK, FoodCORE Team. Improving response to foodborne disease outbreaks in the United States: Findings of the Foodborne Disease Centers for Outbreak Response Enhancement (FoodCORE), 2010-2012. J Public Health Manag Pract. 2015;21(4):E18-26. Available from: https:// www.ncbi.nlm.nih.gov/pubmed/24983761.

3. Seto EY, Soller JA, Colford JM Jr. Strategies to reduce person-to-person transmission during widespread Escherichia coli O157:H7 outbreak. Emerg Infect Dis. 2007;13(6):860-6. Available from: http://wwwnc.cdc.gov/eid/ article/13/6/06-1264_article.

4. Chen T, Leung RK, Zhou Z, Liu R, Zhang X, Zhang L. Investigation of key interventions for shigellosis outbreak control in China. PLoS ONE. 2014;9(4):e95006. Available from: http://journals.plos.org/plosone/article?id=10.1371/ journal.pone.0095006.

5. van de Venter E, Oliver I, Stuart JM. Timeliness of epidemiological outbreak investigations in peer-reviewed European publications, January 2003 to August 2013. Euro Surveill. 2015;20(6).

6. BC Centre for Disease Control. Communicable diseases prevention - Communicable Diseases Prevention and Control Services (CDPACS) is an integrated mix of programs [Internet]. Vancouver: BCCDC [cited 2016 Nov 4]. Available from: http://www.bccdc.ca/our-services/service-areas/ communicable-diseases-prevention.

7. Taylor M, Galanis E. Establishing criteria to initiate enteric outbreak investigations in British Columbia. Can Comm Dis Rep [Internet]. 2014 [cited 2016 Nov 4];40(Suppl 1):10-6. Available from: http://www.phac-aspc.gc.ca/publicat/ccdrrmtc/14vol40/dr-rm40s-1/dr-rm40s-1-interv-eng.php.

8. Centers for Disease Control and Prevention. FoodCORE Salmonella, Shiga toxin-producing E. coli, and Listeria metrics rationale and intent [Internet]. Atlanta (GA): CDC [updated 2016 Feb 17; cited 2016 Nov 4]. Available from: http://www.cdc.gov/foodcore/pdfs/ssl-rationale-intent.pdf.

9. Centers for Disease Control and Prevention. FoodCORE model practice: initial case-patient interviewing [Internet]. Atlanta (GA): CDC [updated 2014 Sep 30; cited 2016 Nov 4]. 
Available from: http://www.cdc.gov/foodcore/pdfs/247981_ foodcore-patient-interviewing_508.pdf.

10. Centers for Disease Control and Prevention. FoodCORE model practice: laboratory timeliness and completeness [Internet]. Atlanta (GA): CDC; [2014 Mar; cited 2016 Nov 4]. Available from: http://www.cdc.gov/foodcore/pdfs/245823_ foodcore-model-practice-factsheet_508.pdf.

11. Centers for Disease Control and Prevention. About FoodCORE [Internet]. Atlanta (GA): CDC; 2014 [updated 2014 Sep 26; cited 2016 Nov 4]. Available from: https:// www.cdc.gov/foodcore/about.html.

12. Centers for Disease Control and Prevention. CDC FoodCORE year one cumulative metrics data - Salmonella, Shiga toxin-producing Escherichia coli, and Listeria (SSL). Report period: October 1, 2010 to September 30, 2011 [Internet]. Atlanta (GA): CDC; [updated 2013 Aug 16; cited 2016 Nov 4]. Available from: http://www.cdc.gov/foodcore/ metrics/metrics-table-year-1-ssl.html.
13. Centers for Disease Control and Prevention. CDC

FoodCORE year three cumulative metrics - Salmonella, Shiga toxin-producing Escherichia coli, and Listeria (SSL). Data report period: January 1, 2013 to December 31, 2013 [Internet]. Atlanta (GA): CDC; [updated 2016 Feb 17; cited 2016 Nov 4]. Available from: http://www.cdc.gov/foodcore/ metrics/metrics-table-year-3-ssl.html.

14. Centers for Disease Control and Prevention. CDC FoodCORE year two cumulative metrics - Salmonella, Shiga toxin-producing Escherichia coli, and Listeria (SSL). Data report period: October 1, 2011 to December 31, 2012 [Internet]. Atlanta (GA): CDC; [updated 2016 Feb 17; cited 2016 Nov 4]. Available from: http://www.cdc.gov/foodcore/ metrics/metrics-table-year-2-ssl.html.

15. Bonacic Marinovic A, Swaan C, van Steenbergen J, Kretzschmar M. Quantifying reporting timeliness to improve outbreak control. Emerg Infect Dis. 2015;21(2):20916. Available from: http://wwwnc.cdc.gov/eid/ article/21/2/13-0504_article.

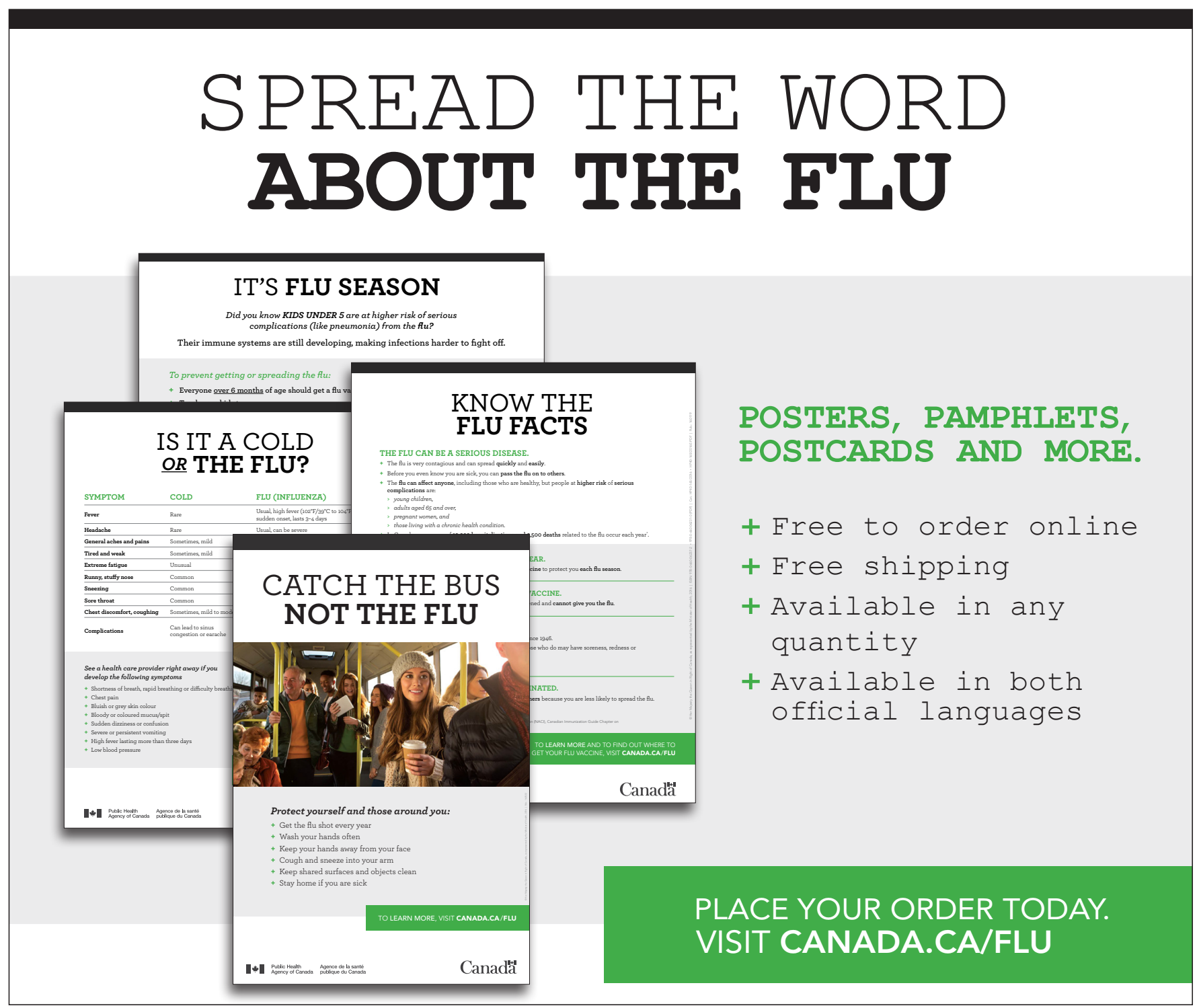

